
\title{
25 Research Sourere \\ Using Pharmacist-Led Tele-Consultation to Review Patients with Chronic Obstructive Pulmonary Disease
}

\section{Wisam Tatari ( $\nabla$ wisam.tatari@nhs.net)}

Drugs4Delivery pharmacy, Tatari Realty Company https://orcid.org/0000-0003-3426-383X

\section{Julie Sowter}

University of Bradford

Khaled Assi

University of Bradford

\section{Research Article}

Keywords: Remote tele-consultation (TC), Pharmacist-Led, Chronic obstructive pulmonary disease (COPD), COVID-19, Review Patients

Posted Date: March 26th, 2021

DOI: https://doi.org/10.21203/rs.3.rs-329733/v1

License: (a) (1) This work is licensed under a Creative Commons Attribution 4.0 International License. Read Full License 


\section{Abstract}

Background: Remote tele-consultation (TC) is seen as one possible solution to circumvent accessibility issues and the feeling of isolation when individuals suffering with Chronic obstructive pulmonary disease (COPD) are at home. Since COVID-19, shifting clinical care to TC has been accelerated.

Objective: A feasibility study was conducted with patients randomized to the intervention, a teleconsultation, or a conventional in-person consultation. The study aimed to inform a future definitive trial to test the impact of pharmacist interventions via TC amongst patients with COPD.

Setting: Conducted in a single community pharmacy by one specialist respiratory pharmacist.

Method: Participants were $>35$ years, with clinician diagnosed COPD and able to communicate in English. Participants were randomized (1:1) to receive an in-person consultation (IPC) or TC after spirometry, oximetry, and BMI measurements in-person. Consultations comprised of COPD review, education and medication optimization. After 6-months, all participants were reassessed in-person.

Main Outcome Measure: Determining the feasibility of the rate of patient recruitment, retention, acceptability of TC, practicalities and completeness of data collection for outcome measures used to assess COPD control and management.

Results : Forty-eight patients from 16 GP practices were recruited over 4-months; 41 (85\%) completed the study. At follow-up, $29 \%$ of participants in the TC group declared a preference for IPC. Collection of data on all outcomes were completed with no adverse events.

Conclusion: The recruitment target was met by employing additional strategies and achieved a satisfactory retention rate. Acceptability of the process of conducting TCs and the completeness of data collection was confirmed.

\section{Impact On Practice}

- TCs are supplemental to a pharmacists' consultation armory. It can be used to access harder-toreach patients who may otherwise have been deprived of pharmaceutical care. The pharmacist may still deem that an IPC is required to help the patient further.

- Approximately two-thirds of patients were open to having a TC in this study.

- TC offers the visual advantage over a telephone consultation and saves time and money over domiciliary visits.

- TC can be used effectively to deliver an assessment of the appropriateness of patients' medication and/ device. It is feasible to counsel on correct inhaler technique, adherence, trigger avoidance and healthy living.

- The simple validated tool, CAT (COPD Assessment Test), which measures COPD control, can be administered via TC. 
- A COPD management plan can be made and emailed to the patient. TC can be particularly useful during an exacerbation or between GP visits.

- TC can be used to signpost to other services such as pulmonary rehabilitation, smoking cessation and vaccinations.

- Any recommendations and/ interventions made could be sent to the GP via the secure NHSmail system. If the GP then acts on the recommendation, a COPD-specific MUR/NMS could be conducted via telephone in order to help embed the information they received and check they are adherent to their treatment regimen.

- The clinic requires read-write access to patients' shared care records and a pathway for prescribing community pharmacists to utilize their prescribing skills which may reduce the number of referrals made to GPs.

\section{Introduction}

In the UK, COPD is regarded as one of the most expensive diseases to treat and it accounts for more time off work than any other condition. ${ }^{1}$ It is essential to explore various approaches to managing COPD, given its impact on Health-Related Quality of Life (HR-QoL) and the considerable drain on healthcare systems. ${ }^{2}$ Ideally, these approaches should comprise of interventions that retard the progressive nature of the disease, achieve a better quality of life ( $Q \circ \mathrm{L})$ and have significant cost savings. ${ }^{2}$ Additionally, immobility and social isolation are important factors which need to be addressed if we wish to provide accessible healthcare to patients with COPD.

The use of assistive technology to offer consultations via remote telecommunication or teleconsultations (TC) is seen as one possible solution to circumvent accessibility issues, the feeling of isolation and vulnerability when individuals suffering with COPD are at home. ${ }^{3}$ Since the COVID-19 pandemic, the use of TC visits has been accelerated worldwide in a bid to curb the disease's spread. Many insurers emphasize that telephone consultations are not a replacement for an In-Person Consultation (IPC) where examination is indicated and thus co-location of patient and practitioner is usually necessary. ${ }^{4}$ This is in contrast to community pharmacists, who although often required to assess patients' symptoms as part of their provision of pharmaceutical care, are rarely involved in physical examinations, such as chest palpation. Thus, it seems feasible for pharmacists, who may otherwise only access these patients via telephone or a rare domiciliary visit, to use TCs when advising immobile patients. Thus, TC will further improve patients' accessibility to community pharmacists.

In principle, TCs offer the visual advantage over telephone communication and save time and costs of travel that domiciliary visits inevitably incur. Thus, quality research is required to comprehensively understand the practicalities as well as any implications of using TCs in a community pharmacy setting. Previous studies implementing a COPD service within community pharmacy have demonstrated favorable financial and clinical benefits to patients. ${ }^{5-7}$ 


\section{Aim of the Study}

A feasibility study was conducted with patients randomized to the intervention, a tele-consultation, or a conventional in-person consultation, in a community pharmacy-based COPD clinic. The study aimed to focus on the rate of patient recruitment, retention, acceptability of TC and practicalities and completion of data collection for outcome measures used to assess COPD control and management.

\section{Ethics Approval}

The study proposal for this research was reviewed by the South Central - Berkshire Research Ethics SubCommittee, who gave a favorable ethical opinion on 15th July 2016.

\section{Methods}

The feasibility study was a six-month, prospective, randomized-controlled, open-label trial conducted in a single community pharmacy by one pharmacist who had previously worked in secondary care as a specialist respiratory pharmacist (SRP) (community pharmacist with qualification in respiratory therapy). Patients were recruited from the pharmacy customer base and local advertisements. Often, 30-50 participants are sufficient for a feasibility study; this study aimed to retain 20 participants per group at follow-up and in keeping with similar studies, allowed for a projected $\mathbf{2 0 \%}$ attrition rate, therefore, $\mathbf{4 8}$ participants (24 per group) was the sample size required.

The inclusion criteria comprised of participants with clinician-diagnosed COPD who were over the age of 35 and able to communicate in English. Since all participants were assessed within the pharmacy's consultation room at baseline (pre-clinic (PC)) and at 6-months, they needed to be able to access the pharmacy. Patients were excluded if they were not responsible for taking their own medications or if they were experiencing or recovering from an exacerbation.

Participants were randomly allocated to the IPC or TC arm of the study. In the TC arm, the consultation took place via Skype ${ }^{\mathrm{TM}}$ or other alternative video chat applications the patient was accustomed to. In such cases, the pharmacist was located in their consultation room and the patient based at their abode (after their PC). In the IPC arm, consultations were conducted within the community pharmacy consultation room where the patient and pharmacist met in person. Both groups received a COPD medication review from a SRP, comprising education on COPD and medication, adherence, inhaler technique, as well as appropriateness of current inhaler/s. Data collection also included acceptability of TC, HR-QoL and cost. All patient information subsequent to baseline was shared with a second pharmacist who documented their recommendations to substantiate whether a community pharmacist without specialist training in respiratory medicine would make the same recommendations. The second pharmacist was blinded to the patient's allocated group. Participants in both the IPC and TC groups were followed up after six months in-person by the investigating pharmacist. 


\section{Results}

\section{Recruitment}

Forty-eight patients were recruited from 16 GP practices over a 4-month period from September to December 2016; 25 (52\%) from the pharmacy's customer base and the remaining 23 (48\%) through advertisements in several local newspapers. The sample consisted of $25(52 \%)$ males and $23(48 \%)$ females. One (2.1\%) was a never smoker, 28 (58.3\%) were ex-smokers and 19 (39.6\%) were current smokers. Participants had a mean COPD Assessment Test (CAT) score of 25.

\section{Retention}

Forty-one participants completed the 6-month follow up consultations which took place between March and June 2017; 21 from the IPC group and 20 from the TC group (85\% retention).

\section{Acceptability}

The process of randomizing patients proved acceptable to patients. All participants completed the acceptability questionnaire. It was determined that $41.7 \%$ of participants in the IPC group would prefer IPC over TCs (see Fig. 1). This was significantly lower in the TC group, at $29.2 \%$, after having experienced its convenience during the study.

\section{Practicalities of TC}

Ten participants (42\%) in the TC group did not have a facility to access Skype ${ }^{\mathrm{TM}}$, so a tablet device was either delivered to their home or given to them after the PC. This was later collected by the pharmacy driver.

On average, the estimated set-up time required for a TC, was an additional 10-minutes per participant, which included how to use the Skype ${ }^{\text {TM }}$ application. During the TCs, the transmission was interrupted on 3 separate occasions (12.5\%). During one of the 3 interruptions, the TC had to be rescheduled.

\section{Outcome Measures and Data Collection}

Completeness of data collection for outcome measures is summarized in Table 1 below. There were no adverse events. It took one-month to analyze the data. 
Table 1

Completeness of data collection for outcome measures

\begin{tabular}{|llll|}
\hline Outcome measure & Assessment tool & \multicolumn{2}{l|}{ Completion rate } \\
\hline COPD control & & IPC & TC \\
\hline Lung function & CAT & $100 \%$ & $100 \%$ \\
\hline Exacerbations & Patient disclosed information & $100 \%$ & $100 \%$ \\
& Checked PMR for rescue medication & & $100 \%$ \\
\hline Hospital admissions & Patient information disclosed & $100 \%$ & $100 \%$ \\
\hline HR-QoL & EQ-5D-5L & $100 \%$ & $100 \%$ \\
\hline COPD management & ABCD assessment tool & $100 \%$ & $100 \%$ \\
\hline Adherence & MARS & $100 \%$ & $100 \%$ \\
\hline Inhaler technique & Checklists & $100 \%$ & $100 \%$ \\
\hline
\end{tabular}

CAT, COPD Assessment Test; EQ-5D-5L, European Quality of Life Five-Dimension Questionnaire; IPC, InPerson Consultation; MARS, Medication Adherence Report Scale; PMR, Patient Medication Record; TC, Tele-Consultation.

There were 185 recommendations made in total, 83 of which required a prescriber to make the intervention. Of these, $24 \%$ were acted upon by the prescriber. Inhaler technique was successfully carried out during TC, however, there were two instances during TC where the patient was out of view of the camera and was asked to re-demonstrate. The outcome measures were successfully administered in both groups and their completeness was checked during the consultation.

Lung function was measured using spirometry, which from a practical perspective, had to be conducted in-person (during PC) on all patients, as the pharmacist needed to instruct the patient on the use of this piece of equipment. The number of exacerbations and hospital admissions were based on patient recall, which may not have been accurate.

In both groups, the number of participants visiting a pharmacy increased during the study period, however, the number of GP visits decreased. The costs increased when comparing the period 6-months prior to the study with the study period (by $\sim 25$ per participant). However, the study would have projected to cost $£ 966.20$ (16.2\%) or $£ 925.80$ (15.5\%) more if domiciliary visits took place ahead of the IPCs or TCs, respectively.

\section{Discussion}


Given that this was a feasibility study, the sample size is often a pragmatic balance between information needs and resources. ${ }^{8}$ Typically, up to 40 patients are recruited for a feasibility study. ${ }^{9}$ Recruitment of Drugs4Delivery patients was achieved via purposive sampling of those who were prescribed inhalers. In order to avoid the risk of not capturing eligible patients, all patients on inhalers were asked their diagnosis via telephone before inviting them to the study. Future studies could use a similar recruitment strategy by using their Patient Medication Record filter to identify all the pharmacy patients that use inhalers.

The most notable challenge of this research study was recruiting the required number of patients from a single community pharmacy's patient base. A drawback of this study was that just 25 patients could be recruited via this method. This was overcome by adapting the approach half way through, via advertising more widely in local newspapers. Thus, the recruitment target was met. The advertising strategy mitigated against the limitations of the initial recruitment strategy and this approach could be incorporated from the outset in any larger, future studies. In addition, those participants that did not turn up to their baseline consultation, attended after they were re-scheduled.

In this study, participants recruited via advertising were either keen to be involved in research or were keen to consult a SRP and learn whether there were other treatment options available to them. The lack of referrals from general practice could potentially be circumvented by presenting the study proposal, inperson, to all the local surgeries and involving clinicians at an earlier stage in the research design. Future studies should be mindful that this could result in selection bias as participation in studies is usually accepted by motivated patients.

\section{Patient Retention}

A high retention rate was achieved in the study. A source of bias for prospective studies is the potential for dropouts, which was low in this study, as significant efforts were made to avoid this. These included, negotiating treatment decisions with the patient and conducting the clinics in a professional setup as advocated by Ali Murshid and Mohaidin. ${ }^{10}$ Other factors that may have contributed to the low number of participants being lost to follow-up could have been existing rapport with Drugs4Delivery patients and informing participants that they would be reassessed in 6-months' time in order to determine any change in their condition. This latter information may have motivated them to find out about whether they had gained any benefits from the interventions after 6-months.

These strategies could be used to inform the design of future multi-centered studies in order to minimize attrition, although recruiting via the pharmacy's patients should be interpreted with caution, as a larger study involving multi-centers may vary from this single-site study. Although, the multi-site approach improves recruitment and retention potential compared to single site, factors such as managing research, ensuring consistent processes and high-quality data, and having clear communication processes between researchers/clinicians, become crucial. These issues require a significant amount of planning and meetings for each research site, which involves going through protocol, standard operating procedures and processes. ${ }^{11}$ 


\section{Acceptability of Tele-Consultations}

An anonymized feedback questionnaire was administered to each participant (in both groups) in order to offer their opinions of the clinic. From the questionnaire used, it was determined that participants in the TC group were more receptive to using TC after having experienced its convenience during the study (see Table 1). It was thought that the older participants would be resistant to TC. However, this study found they became more amenable once they had been supported through the process of using it. From the study, it was evident that patients generally felt more comfortable talking from within the comfort of their home than in-person within a consultation room. Patients commented on the value of having a TC, especially during an exacerbation or for immobile patients. This was supported by Vitacca, Montini and Comini ${ }^{12}$ who reported that the best outcomes came from TCs dedicated to more severe cases, frequent exacerbators with multimorbidity and limited community support. Thus, selecting these ideal candidates could be the key to optimizing the use of TC in future RCTs.

The research literature on TCs is sparse but has begun to accumulate. ${ }^{13}$ Sorknaes et al. ${ }^{14}$ reported high patient satisfaction, which was also reported in other earlier studies. ${ }^{15-17}$ Although these studies had a similar demographic, Rahimpour et al. ${ }^{16}$ and Whitten and Mickus ${ }^{17}$ also included patients with congestive heart failure. Tatari ${ }^{18}$ reported that $12 \%$ of patients experiencing TCs for the first time thought they would benefit more from an IPC than a TC and $9.5 \%$ did not consent to having a TC, as they preferred to have an IPC; however, this was not specific to COPD patients, and participants were from a younger demographic on average.

Of the 185 patients screened during this feasibility study, only 8 (4.5\%) specifically gave the reason of not wanting to use TC, however, this could have been more since 35 patients did not give a reason for declining to participate. Offering a tablet device to those without Skype access may have led to a higher acceptance rate. Participants in a study by Gund et al. ${ }^{19}$ also reported positive experiences with TCs, and patients found the technology easy to use and better than ordinary phone calls, although, this was not always the case with motivating staff.

There were some notable differences experienced between the two groups; most notably, during an IPC when the patient smelt of smoke, it was likely that they were either a smoker or they experienced passive smoking. However, this could not be picked up when conducting TCs. Furthermore, for TC to be successful, a clear uninterrupted transmission is required, a significant limitation of this medium. Losing transmission means the consultation cannot take place and must be rescheduled. From the pharmacist's perspective, organizing the IPC was a great deal easier than the TC, especially if the participant did not have TC access or did not know how to work it. IPCs are the norm for patients when seeing their healthcare professional, whereas TC seemed overwhelming if they were new to the concept.

\section{Practicalities of study implementation}


This study sent recommendations via postal mail for both groups and lack of uptake (approximately a quarter were actually implemented) brings into question whether some recommendations actually reached the prescriber. Until read-write access to patient notes is implemented, future studies could try alternative communication channels such as phoning the practice pharmacist or emailing via NHSmail (this option was not available at the time of the study).

In a bid to improve uptake of recommendations, there were occasions where the SRP empowered patients to take the recommendations forward with their GP/RNS. This appeared to improve uptake of interventions in several instances, however, communicating via patients is not the recommended pathway. In these scenarios, the SRP felt frustrated for the patient, and despite having a prescribing qualification, there is no current pathway to prescribe an alternative inhaler device on the NHS in this setting. Future studies should focus on building stronger relationships with local surgeries and obtaining read-write access for the pharmacist to inform their recommendations.

During the study, similar numbers of patients from the TC group quit tobacco smoking compared to the IPC group, which suggests that TC was not a barrier in this regard. Only one person was recommended oxygen therapy by the pharmacist due to their oximetry readings and later went on to commence therapy. Pulmonary rehabilitation is a key non-pharmacological component that shows improvement in QoL in patients with COPD. ${ }^{20}$ There were referrals to pulmonary rehabilitation either directly or via the GP. Directly referring patients achieved a $100 \%$ success rate in attendance, whereas only a third attended via the GP.

During the PC of this current study, the appropriateness of patient's inhaler device(s) was assessed using the In-Check DIAL meter. This device helps to establish the suitability of the inhaler device based on the patient's inspiratory force capabilities. If their inhaler device was inappropriate, an alternative inhaler device was suggested to their GP. A barrier to TCs was in the assessment of patients' inspiratory force using this device; from a practical perspective, this measurement had to take place in-person for all participants, as the pharmacist needed to instruct the patient on its use.

The results in this study provide some assurance that TCs are not a barrier for pharmacists to making interventions/recommendations when compared to IPCs and give an indication of what can be expected in future studies. These findings are of importance, particularly when reviewing immobile patients, who are harder-to-reach and often deprived of pharmaceutical advice. To enable an all-inclusive NHS, pharmacists who may otherwise only access these patients via telephone or a rare domiciliary visit, could use TCs with its visual advantage over telephone communication and bearing in mind the time and costs of travel that domiciliary visits inevitably incur (see Fig. 2).

As a SRP, the researcher conducted the consultations and mitigated against potential sources of bias by concealing the randomization of participants, as well as using validated and objective tools wherever possible. The process of randomization into the respective groups proved acceptable to patients, thus will be feasible to conduct in a future definitive trial. Employing an independent SRP to conduct these clinics or analyse the data would be ideal if funding was not a barrier. This would also reduce the risk of 'vested 
interest bias' that could be attributed to the researcher. It is crucial to mitigate against the risks that ensue in having an insider researcher. Also, it was not possible to blind the SRP or the participant to which group they were allocated (open-labelled trial). This issue was mitigated against by keeping systematic differences between the two groups to a minimum (performance bias); this was achieved by observation by another pharmacist and standardizing the consultations of the two groups, where the only major variability was conducting the consultation via IPC or via TC.

\section{Conclusion}

COPD is a significant cause of morbidity and mortality and also represents a high economic and social burden internationally. Since patients with COPD can become housebound, they are less likely to have their medication reviewed by their pharmacist. This feasibility study conducted from a single pharmacy with a SRP, identified constraints with reliance on the pharmacy's customer-base to recruit a sufficient sample size, but demonstrated that this could be supplemented via local advertisements. The study achieved a satisfactory retention rate and confirmed acceptability of the process of conducting TCs and the completeness of data collection. Although, lost transmission and the patient being out of view of the camera were practical barriers to the success of TC.

\section{References}

1. Keehan SP, Stone DA, Poisal JA, et al. National Health Expenditure Projections, 2016-25: Price Increases, Aging Push Sector To 20 Percent Of Economy. Health Aff. 2017;36:553-563.

2. Global Initiative for Chronic Obstructive Lung Disease. Global Strategy for the Diagnosis, Management and Prevention of COPD. 2017. Available at: http://goldcopd.org/gold-2017-globalstrategy-diagnosis-management-prevention-copd/

3. Mathar H, Fastholm P, Sandholm N. A qualitative study of televideo consultations for COPD patients. J. Nurs. 2015;24:205-209. https://doi.org/10.12968/bjon.2015.24.4.205

4. McLean S, Chandler D, Nurmatov U, Liu J, Pagliari C, Car J, Sheikh A. Telehealthcare for asthma. Cochrane Database Syst. Rev. https://doi.org/10.1002/14651858.CD007717.pub2

5. Tommelein E, Mehuys E, Van Hees T, et al. [Effectiveness of pharmaceutical care for patients with COPD: translated review of the recently published PHARMACOP trial]. Pharm. Belg. 2014;4-14.

6. van Boven JFM, Stuurman-Bieze AGG, Hiddink EG, et al. Effects of targeting disease and medication management interventions towards patients with COPD. Med. Res. Opin. 2016;32:229-239.

7. Wright $D$, Twigg $M$, Barton $G$, et al. An evaluation of a multi-site community pharmacy-based chronic obstructive pulmonary disease support service: Community pharmacy-based COPD service. J. Pharm. Pract. 2015;23:36-43.

8. Eldridge SM, Lancaster GA, Campbell MJ, et al. Defining Feasibility and Pilot Studies in Preparation for Randomized Controlled Trials: Development of a Conceptual Framework. PLOS ONE 11. 2016. e0150205. 
9. Craig P, Dieppe P, Macintyre S, Michie S, Nazareth I, Petticrew M. Developing and evaluating complex interventions: the new Medical Research Council guidance. BMJ. 2008;337, a1655.

10. Ali Murshid M, Mohaidin Z. Models and theories of prescribing decisions: A review and suggested a new model. Pract. 2017;15:990-990.

11. Lijmer JG, Mol BW, Heisterkamp S, et al. Empirical evidence of design-related bias in studies of diagnostic tests. JAMA. 1999; 282:1061-1066.

12. Vitacca M, Montini A, Comini L. How will telemedicine change clinical practice in chronic obstructive pulmonary disease? Therapeutic Advances in Respiratory Disease. 2018. 12. https://doi.org/10.1177/1753465818754778

13. Armfield NR, Bradford M, Bradford NK. The clinical use of Skype-For which patients, with which problems and in which settings? A snapshot review of the literature. I J. Med. Inf. 2015;84:737-742.

14. Sorknaes AD, Madsen H, Hallas J, Jest P, Hansen-Nord M. Nurse tele-consultations with discharged COPD patients reduce early readmissions-an interventional study. Respir. J. 2011a;5:26-34.

15. Mair FS, Goldstein P, May C, et al. Patient and provider perspectives on home telecare: Preliminary results from a randomized controlled trial. Telemed. Telecare. 2005;11:95-97.

16. Rahimpour M, Lovell NH, Celler BG, McCormick J. Patients' perceptions of a home telecare system. J. Med. Inf. 2008;77;486-498.

17. Whitten P, Mickus M. Home telecare for COPD/CHF patients: outcomes and perceptions. Telemed. Telecare. 2007;13:69-73.

18. Tatari W. How Skype can enable pharmacists to help more patients. Pharmacy Voice. 2016;1(2):6

19. Gund A, Sjöqvist BA, Wigert H, Hentz E, Lindecrantz K, Bry K. A randomized controlled study about the use of eHealth in the home health care of premature infants. BMC Med. Inform. Decis. Mak. 2013;13. https://doi.org/10.1186/1472-6947-13-22

20. Lacasse Y, Goldstein R, Lasserson TJ, Martin S. Pulmonary rehabilitation for chronic obstructive pulmonary disease, in: The Cochrane Collaboration (Ed.), Cochrane Database of Systematic Reviews. John Wiley \& Sons, Ltd, Chichester, UK. 2006.

\section{Figures}




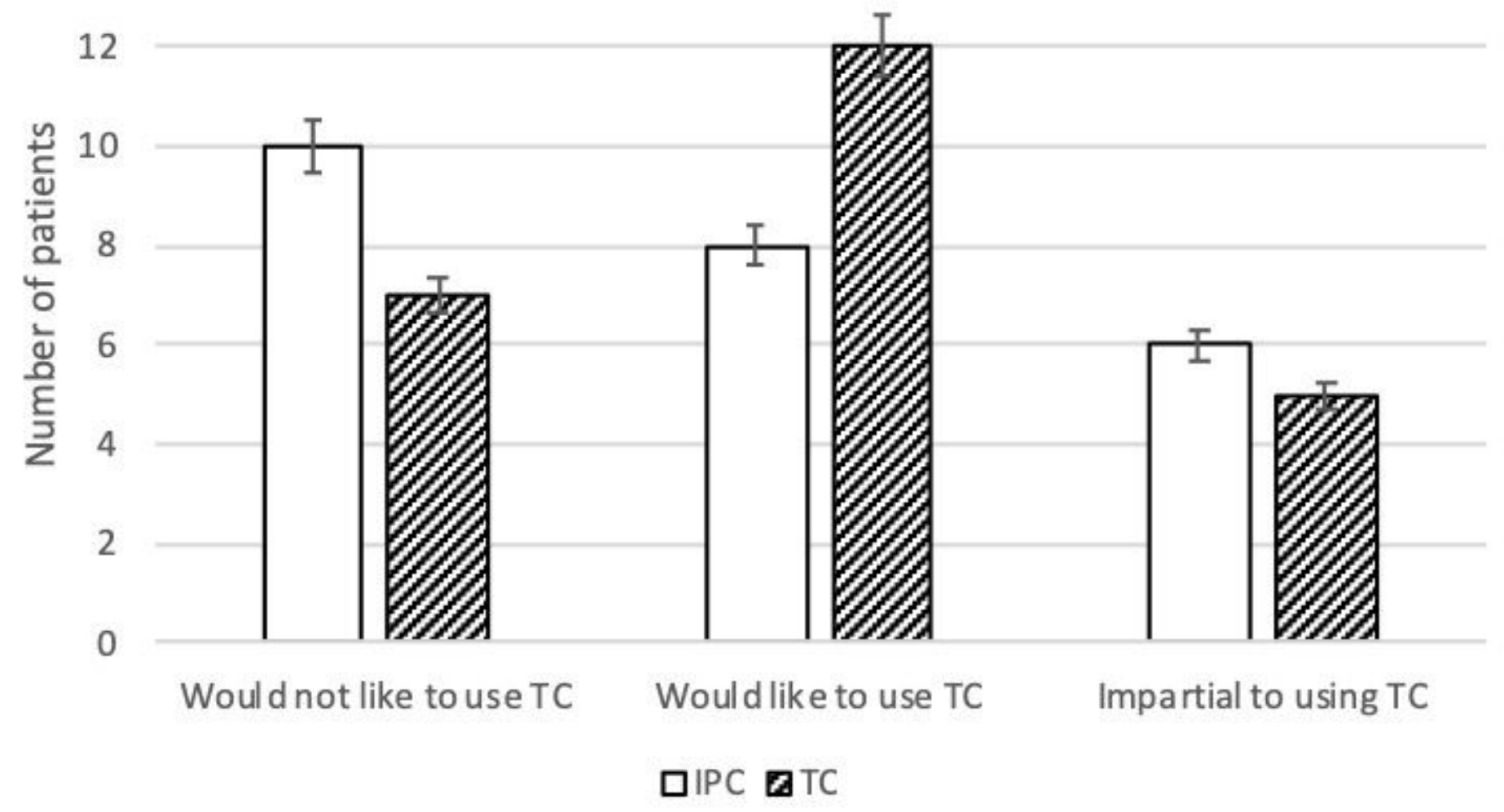

Figure 1

The number of participants within each group who were willing, unwilling or impartial to using TC in the future. IPC, In-Person Consultation; TC, Tele-Consultation. 


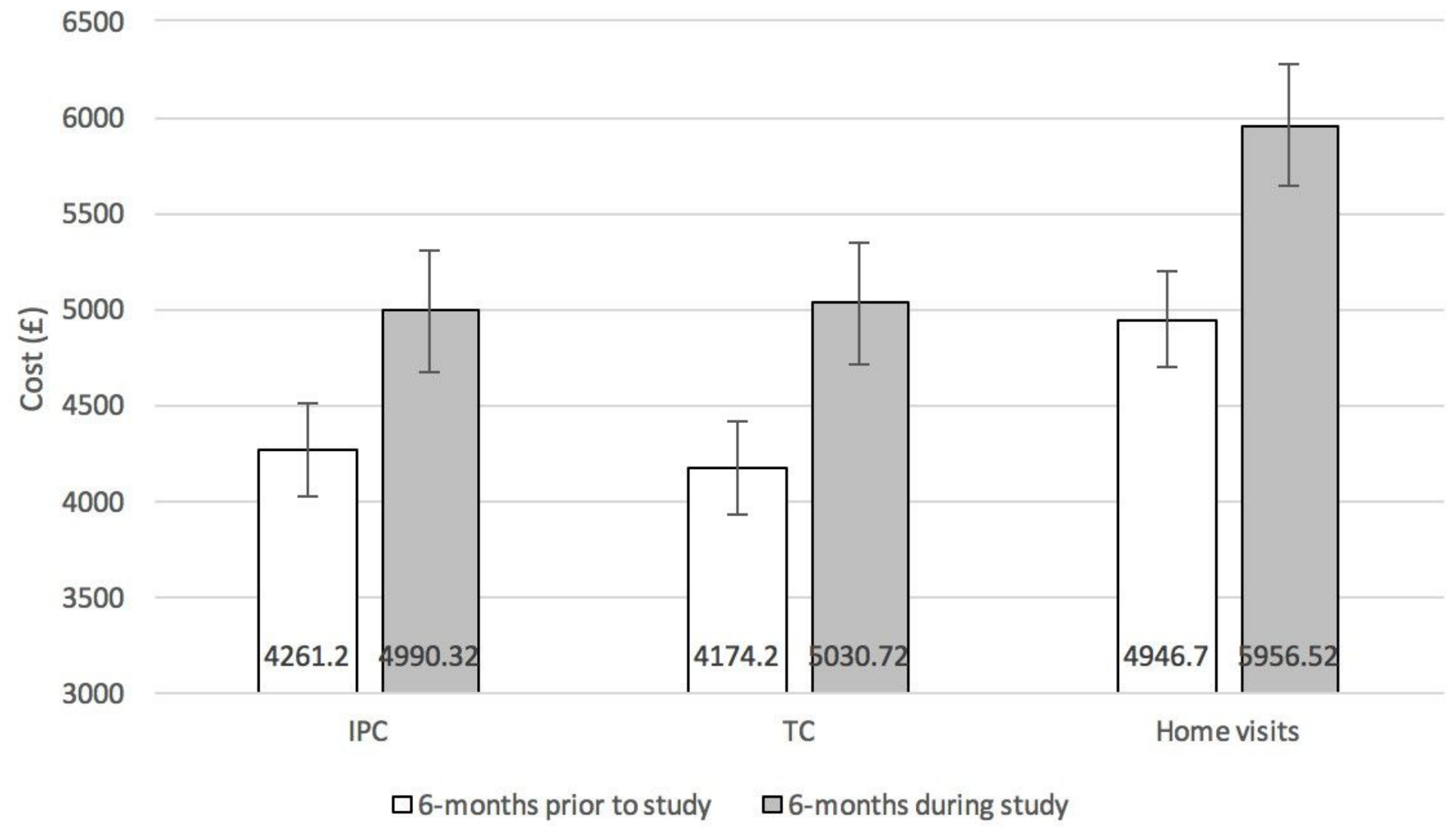

Figure 2

The total NHS costs on the different consultation methods 6-months prior to the study and 6-months during the study. IPC, In-Person Consultation; TC, Tele-Consultation. 